\title{
Infección del tracto urinario
}

\author{
Rosanna Lagos Z. ${ }^{1}$; Inés Bravo L. ${ }^{2}$
}

\section{Urinary tract infections}

\begin{abstract}
During the past ten years, new concepts on host-parasite interaction have allowed better understanding of the pathogenesis of urinary tract infections (UTH). In addition, significent progress has been achieved with the development of new methods for differentiating upper and lower UTI. This review is Intended to discuss current information about virulence factors of Escherichia coli, host genetic risk markers associated to recurrence and complications, and new diagnostic too is for localizing the anatomic place of urinary tract infections.

(Key words: urinary tract infections, $E$. coli, virulence factors, host factors.)
\end{abstract}

El término infección del tracto urinario (ITU) comprende un grupo heterogéneo de entidades clínicas acompanadas de bacteriurja y leucocituria, cuyos pronósticos y manifestaciones sintomátjcas varían de acuerdo al sitio anatómico comprometido. La importancia epidemiológica de estas enfermedades queda en evidencia en numerosos estudios extranjeros que demuestran su alta incidencia, particularmente en mujeres $y$ nifíos. Asimismo, y aun de mayor relevancia, es que los pacientes con ITU que compromete el parénquima renal -pielonefritis aguda (PNA)están expuestos a elevado riesgo de recurrencias, cicatrices renales e hipertensión arterial: aunque la insuficiencia renal crónica es una complicación rara, la elevada incidencia de ITU determina que estas enfermedades figuren entre las primeras causas de insuficiencia renal terminal, a cualquier edad.

En consideración a estos hechos, la investigación básica y clínica en este campo se ha dirigido hacia la detección de elementos que condicionan - se asocian al desarrollo de complicaciones y que permitan identificar, entre el total de indivi. duos con bacteriuria, a aquellos en los que es necesario concentrat mayor cantidad de recursos de atención médica. Esos estudios se han canalizado fundamentalmente al desarrollo de técnicas destinadas a perfeccionar la localización clínica de la infección, identificar marca-

1. Servicio de Pediatría, Hospital Roberto del Río.

2. Universidad de Chile, Departamento de Pediatria, Facultad de Medicina, Campus Norte. dores de riesgo en el huésped e investigar factores de virulencia bacterianos. El propósito de esta revisión es exponer y comentar la información más relevante recopilada durante los últimos 10 años en torno a estos tres aspectos.

\section{Sitio anatomico de la infección}

El diagnóstico de localización de la ITU tjene implicancias en el tratamiento, estudio y pronóstico de la enfermedad. La necesidad de reconocer acertada y precozmente los casos de PNA radica en las evidencias que demuestran la asociación de cicatriz renal, signo no siempre seguro de infección del parénquima, con el desarrollo ulterior de afecciones crónicas. Winberg ${ }^{1}$ estudió la evolución a largo plazo de niños que presentaron PNA complicada con cicatriz renal; sus observaciones ilustran de manera elocuente estas asociaciones: al cabo de 30 años, $23 \%$ de los pacientes presentaban hipertensión arterial, 10\% tenían insuficiencia renal terminal y otro $23 \%$ habían requerido nefrectomia unjlateral. Además, $13 \%$ de las niñas que se embarazaron cur. saron con toxemia severa. En seguimientos del mismo tipo, otros autores han informado de resultados similares ${ }^{2-6}$.

La evaluación de pruebas destinadas a identificar el lugar de la ITU invariablemente tropieza con la carencia de un "patrón de oro" capaz de reconocer todos los casos de infecciones altas. En su defecto, y aunque la cicatriz renal se produce en no más de $10 \%$ de los episodios de 
PNA, los primeros estudios la utilizaron de referencia para analizar la utilidad de signos clíni$\cos y$ de laboratorio, como indicadores de ITU alto, en función de la asociación de aquéllos con el desarrollo de cicatriz renal o como predictores de su aparición. Así, quedó establecido que la fjebre, el dolor lumbar, los signos clínicos de compromiso sistémico y las alteraciones de laboratorio propias de la respuesta de fase aguda -leucocitosis, neutrofilia, aumentos de la velocidad de eritrosedimentación (VHS) y de la proteína $\mathrm{C}$ reactiva (PCR) - son significativamente más frecuentes entre los pacientes con ITU que desarrollan recurrencias y cicatriz renal, ${ }^{7}$. Aunque accesibles, estos parámetros se han de. mostrado insuficientes para detectar todos los casos de PNA, inconveniente que adquiere particular relevancia en nifios pequefios, en quienes la frecuencia de PNA sería subestimada, debjdo a las manifestaciones inespecíficas de la en. fermedad ${ }^{9}$. Por otra parte, estudios clínjicos ${ }^{10} y$ experimentales ${ }^{11}$ demuestran que la vulnera. bilidad renal a la agresión de las pielonefritis aumenta en forma directamente proporcional con la tardanza del tratamiento e inversamente con la edad, pues la probabilidad de desarrollar cicatriz renal alcanza su máximo durante el primer aflo de vida y decrece muy significativa. mente después de los siete años ${ }^{\mathbf{1 2}}$ Por su parte, la edad es una determinante crítica en el dafo funcional renal secundario a ITU ${ }^{13}$.

En base a las alteraciones funcionales obser. vadas durante la PNA, se ha sugerido que la capacidad de concentración renal puede ser utilizada como prueba específica y sensible en el diagnóstico diferencial de infecciones altas $y$ bajas. El método de estudio fue simplificado por Aronson ${ }^{14}$, quien señaló que los inconvenientes y riesgos de la prueba de concentración se han reducido notablemente gracias a la disponibilidad de un análogo sintético de la hormona autidiurética (I-deamino-8-D-arginina vasopresina, DDAVP).

Más recientemente, la cintigrafía con ácido dimercaptosuccínico marcado con tecnecio (TcDMSA), basada en la afinidad de este compuesto por las células tubulares, ha sido propuesta como un examen de alto rendimiento, tanto en el diag. nóstico de localización de la ITU como en la pesquisa de lesiones cicatrizales. Los resultados de dos importantes estudios experimentales ${ }^{15,16}$ coinciden en demostrar que, durante la fase aguda de la enfermedad, es posible observar zonas hipocaptantes en más de $85 \%$ de los casos de PNA histológicamente comprobados. En el terreno clínico, se ha señalado que la disminución global de captación y diferencias de captación superiores a $12 \%$ entre ambos rifiones reflejan la existencia de áreas de isquemia y que estos hallazgos se encuentran fuertemente asociados a los signos clásicos de $\mathrm{PNA}^{17}$. De manera semejante, después de cursada la infección, la sensibilidad del Tc-DMSA para detectar lesiones cicatrizales superaría a la del estudio radiológico convencional, reduciendo, además, la irradiación requerida para tal estudjo ${ }^{17,18}$. Como limitaciones del procedimiento se ha señalado que la recuperación de las anomalidades funcionales puede tardar hasta 4 meses $y$, por consiguiente, el diagnóstico de cicatriz renal no puede fundamentarse en el hallazgo de áreas de baja captación cuando el estudjo con DMSA ha sido realizado antes de este plazo ${ }^{17}$. Por otra parte, la correlación con la histopatología renal 15,16 ha revelado que la sensibilidad del cintigrama decrece significativamente cuando la magnitud del compromiso cortical renal es inferior a $15 \%$.

Por último, se ha postulado que la excreción urinaria de enzimas lizosomales traduce infección del parénquima renal, por lo que la presencia de las mismas en la orina podría ser de ayuda en el diagnóstico de la ubicación de la ITU; entre ellas, la N-acetil-B-D-glucosaminidasa (NAG) ha concentrado el interés, debido a que posee características que facilitan su medición ${ }^{19}$. Se ha encontrado eliminación elevada de NAG en pacientes con signos ciínicos y de tabo. ratorio de PNA, en tanto que los valores de la enzima fueron normales en la mayoria de los niños con cistitis ${ }^{20,21}$. Otros autores, por el contrario, observaron excreción aumentada de NAG en $27 \%$ de los niños con signos de infección baja, señalando que tales pacientes tenian evidencja de reflujo vesicoureteral, concluyendo que NAG no es útil para el diagnóstico de localización de la ITU, pero que permitiría iden. tificar pacientes con cistitis que requieren estudio radiológico ${ }^{19}$

\section{Factores de patogenicidad para la vía urinaria}

Puesto que Eschechiria coli es el agente más frecuentemente identificado en ITU, esta revi- 
sión sólo hará mención a la información relacio. nada con este patógeno.

Varios estudios epidemiológicos realizados en niños en la década de los 70 permitieron suponer una correlación entre las caracteristicas de la cepa infectante y la forma de presentación de la ITU. Las cepas de $E$. coll ajsladas de niflas con bacteriuria asintonática eran más frecuente. mente autoaglutinables y sensibles a la actividad bactericida del suero que las recuperadas de pacientes con PNA, y los serotipos OKH se distribuian de manera desigual entre las cepas causantes de PNA, cistitis y colonización del intestino grueso ${ }^{22,23}$. Posteriormente se caracterizaron clones pielonefritogénicos ${ }^{24,25}$, que pertenecen a un número limitado de serotipos $\mathrm{OKH}^{26}$ $y$ de tipos alelomórficos de enzimas ${ }^{27,28}$; frecuentemente poseen resistencia a la actividad bactericida del suero y producen hemolisinas y aerobactinas. Aunque útiles como marcadores epidemiológicos, el rol especifico de estos rasgos fenotípicos en la patogenia de la ITU es difícil de evaluar, puesto que las cepas aisladas frecuentemente coexpresan varios de ellos y los clones asaciados a uno y otro patrón de enfermedad difieren en más de uno de estos rasgos. Por ejemplo, ni la resistencia a la actividad bactericida del suero ni la producción de hemolisinas se correlaciona con la forma clínica de ITU $^{29}$ y la expresión de aerobactinas se encuentra frecuentemente asociada a la producción de adhesinas $\mathrm{P}^{30}$

Svamborg y cols. demostraron que habia correlación directa entre la intensidad de adherencia a células epiteliales y severidad de la ITU $^{31,32}$, lo que ha sido confirmado poste. riormente en pacientes de todas las edades ${ }^{33-35}$. En contraste con los primeros factores mencionados, cuyo mecanismo patogénico es incierto, la adhesión a células de huésped es una fase común e indispensable en la etiopatogenia de mu. chas enfermedades infecciosas; por este motivo, el estudio de las adhesinas ha concentrado gran parte de la investigación en tomo a factores de urovirulencia. El estado actual del conocimiento referente a las principales adhesinas de $E$. coli ha sido resumido en publicaciones recien$\operatorname{tes}^{36,37}$.

Las adhesinas de $\boldsymbol{E}$. coli han sido clasificadas en 3 categorias: tipo $1, \mathrm{P}$ y $\mathrm{X}^{36}$. La expresión de adhesinas tipo 1, caracteristica constante $y$, al parecer, imprescindible para la colonización intestinal, es menos frecuente entre $E$. coli de origen urinario y parece no estar relacionada en forma directa con la patogenia de la ITU. La adhesión dependiente de esta fimbria es mediada por $\alpha$-D-manosa y detectable mediante hemaglutinación manosa-sensible de glóbulos rojos de cobayo.

La adhesina $P$, en cambio, es reconocida como el indicador más relevante y caracteristico de virulencia, en el aparato urinario, de las cepas causantes de PNA. Actualmente se dispone de abundante información sobre la estructura y genética de esta fimbria y se sabe que, en la mayoría de los casos, su receptor específico es la molécula $\alpha$-D-galactosil-(1->4)$\beta$-D-galactopiranosa (Gal-Gal), presente en los glicolípidos de glóbulos rojos y células uroepiteliales de individuos con grupo sanguíneo $\mathrm{P}^{38,}{ }^{39}$. Tambiên se ha documentado que ciertos antigenos del grupo $\mathrm{M}$ podrían actuar como receptores para esta adhesina ${ }^{40}$.

El rol patogénico de la adhesina $\mathrm{P}$ ha sido ampliamente evaluado en función de su asociación con ITU alto ${ }^{33-37,41,42}$. Al respecto, ha habido consenso en reconocer su participación en la génesis de la PNA, presumiblemente favoreciendo la colonización ascendente de las vías urinarias. Además, se ha demostrado que las cepas Gal-Gal positivas inducen una respuesta inflamatoria de mayor intensidad, postulándose que la adhesión a células epiteliales facilitaría la presentación de componentes bacterianos que promueven la respuesta inflamatoria ${ }^{43}, 44$.

Otros estudios han analizado la frecuencia de cicatriz renal $y$ reflujo vesicoureteral en función de este factor de virulencia; en este sentido, los resultados han sido menos coincidentes: después del primer episodio de PNA en varones el desarrollo de cicatriz renal fue significativamente mayor entre los niños infectados por cepas Gal-Gal negativas que entre los pacientes en los que se aisló $E$. coli, productor de adhesinas $\mathbf{P}$, diferencia no explicable sólo por mayor frecuencia de casos con reflujo vesicoureteral en el primer grupo, concluyéndose que, en esta situación, la identificación de una cepa Gal.Gal negativa predice el desarrollo de cicatriz renal y obliga a realizar estudios para investigar reflujo o malformaciones ${ }^{43}$. En trabajos realizados en niñas con PN recurrente también se encontró mayor porcentaje de cepas Gal-Gal negativas entre las pacientes que de. sarrollaron cicatriz renal $1^{41,42}$. En contraste con los resultados citados, otros autores encon. 
traron diferencias en la frecuencia de cepas GalGal negativas aisladas de pacientes con y $\sin$ reflujo o malformaciones de las vías urina$\operatorname{rias}^{36,46,47}$.

En suma, si bien existe consenso en que la adhesina $P$ es un factor decisivo en la virulencia, en la vía urinaria, de $E$. coli y en admitir su rol en la génesis de la PNA, su participación en el desarrollo de cicatriz renal y recurrencia de la infección no ha sido suficientemente esclarecj$\mathrm{da}$; por consiguiente, la utilidad de investigarlas en las cepas de pacientes con ITU está aún en discusión. La importancia de la fimbria $P$ de $E$. coll, como antígeno inmunizador para pre. venir las ITU, ha comenzado a estudiarse recientemente. Los resultados experimentales sugieren esta posibilidad ${ }^{48,49}$ y permiten esperar que durante los próximos años aumente el interés de los investigadores en desarrollo de vacunas.

Otro componente con estructura de fimbria, más recientemente descrita, es la adhesina $F$, muy relacionada genética y morfológicamente con la $\mathbf{P}$, también capaz de producir hemaglutinación en presencia de manosa. Ella "reconoce", en forma específica, al antígeno de Forssman, abundante en los glóbulos rojos de cordero e identificado también en la pelvis renal humana. El receptor para esta adhesina es la galactosa-N-acetil- $\alpha(1->3)$ galactosa-N-acetil y su expresión fenotípica es detectable mediante hemaglutinación manosa-resistente de glóbulos rojos de cordero. La coexpresión de adhesinas $F$ y $P$ es significativamente más frecuente en $E$. coli aislados de pacientes con pielonefritis, que en cepas fecales o de pacientes con ITU bajo $^{36,50}$. La sola presencia de adhesinas pareciera no conferir virulencia para la via urinaria a las cepas de $E$. coli, ya que este rasgo fenotipico aislado sería más frecuente en las bacterias fecales ${ }^{36}$.

Por último, se ha llamado adhesinas $\mathrm{X}$ a un grupo heterogéneo de estructuras sin aspecto de fimbrias, capaces de producir aglutinación manosa-resistente de glóbulos rojos humanos carentes de antígeno P1 y cuyos receptores específicos no han sido identificados ${ }^{36,51}$. A este grupo pertenecen adhesinas dependientes de operones no relacionados entre sí, uno de los cuales, el operón "afal", codifica para una adhesina capaz de conferir adherencia a células uroepiteliales humanas ${ }^{49,52,53}$. Al comparar la distribución fenotípica de adhesinas X y de secuencias gené- ticas homólogas a este operón en cepas fecales, de PNA y de ITU bajo, la frecuencia de cepas afal positivas $y$ productoras de adhesina $X$ es significativamente mayor entre las aisladas de pacientes con cistitis; sin embargo, una proporción importante de las cepas que hibridaron con la sonda genética pasó desapercibida por la prueba de hemaglutinación y viceversa, fenómeno que, según los autores, serja evidencia de la heterogeneidad genética y fenotípica de este grupo de adhesinas ${ }^{36}$. En contraste con la alta frecuencia de coexpresión de las adhesinas $P$ y $F$, no se observó que las adhesinas $X$ se relacionaran con la presencia de otras.

\section{Factores de susceptibilidad en el huésped}

Los eventos subsecuentes a la colonización, y que permiten la infección del uroepitelio, son determinados tanto por la virulencia del germen como por los mecanismos de defensa del huésped. Entre estos últimos, son fundamentales la indemnidad estructural, dinámica y funcional de la vía urinaria, cuyas alteraciones favorecen las ITU aún por gérmenes de poca virulencia. Es así como las uropatías obstructivas, las anomalías funcionales de la vejiga y el reflujo vesicoureteral son factores reconocidamente asociados a las infecciones del tracto urinario, sus recurrencias, tendencia a la formación de cicatrices renales y evolución hacia la insuficiencia renal crónica. Debido a la amplia difusión de que han sido objeto, en esta revisión no se mencionarán estos factores.

Durante la últjma década se ha prestado especial atención a un conjunto de características genéticas del huésped como posibles indicadores de su susceptibilidad a ITU, forma de evolución y complicaciones. Entre ellos, los mejor estudiados han sido los grupos sanguíneos clásicos, el grupo sanguíneo $\mathrm{P}$ y el estado secretor.

Cruz-Coke fue el primero en demostrar una relación entre susceptibilidaci a las ITU y grupo sanguíneo $B^{54}$. Más tarde, Ratmer confirmó esta observación y agregó que este rasgo genético se asacia a infecciones crónicas del riñón ${ }^{55}$, sugiriendo la participación de isoaglutininas en este fenômeno de selección.

Puesto que en el grupo sanguíneo P se han identificado antígenos que son reconocidos de modo específico por las fimbrias $\mathrm{P}$ de $E$. coli varios estudios han analizado su asociación con ITU. 
En uno se encontró 97\% de portadores de grupo P1 entre nifios con pielonefritis recurrente, en una población con $75 \%$ de prevalencia de ese marcador sanguineo ${ }^{56}$. En ot ra agrupación con $31 \%$ de prevalencia de $P 1$, este rasgo genético se detectó en $50 \%$ de los niños con ITU recurrentes y $62 \%$ de los niños con ITU febril ${ }^{5}$. En ambos casos se concluyó que la frecuencia de grupo $\mathbf{P 1}$ es significativamente más alta en individuos con PNA y recurxente, aún en ausencia de reflujo y malformaciones.

El estado secretor se define como la capacidad de secretar formas hidrosolubles de los antígenos de los grupos sanguíneos a los fluidos corporales. Se postula que la presencia de antígenos libres en la orina bloquearía los sitios de unión específica de la bacteria, impidiendo su adherencia a la célula epitelial. Se ha demostrado aumento de la frecuencia de individuos no secretores sólo entre los pacientes con PN recurrente complicada de cicatriz renal ${ }^{41}$. Otros también encontraron esta asociación en pacientes con ITU recurrente, con o sin evidencias de cicatriz renat $^{58} \mathrm{y}$, por último, algunos se refieren a la relación entre estado no secretor e ITU, sin mencionar localización ni complicaciones ${ }^{59}$.

\section{Comentario}

Las infecciones del tracto urinario y sus complicaciones son de alta prevalencia, afectan por igual a individuos de áreas geográfícas y condiciones socioeconómicas diversas $y$, en consecuencia, son un tema de permanente interés para investigadores clínicos y básicos.

En el terreno clínico, los progresos más relevantes conseguidos durante los últimos 10 aftos consisten en el desarrollo de métodos de estudio destinados a precisar la ubicación anatómica de la ITU. Los trabajos experimentales coinciden en indicar que la prueba de concentración renal con DDAVP y el estudio con Tc-DMSA son las pruebas no invasivas más sensibles y especifficas para el diagnóstico de PNA. Pese a ello, las posibilidades de evaluar su utilidad clínica y de comparar el rendimiento de estas pruebas entre sí se ven aún dificultadas por las limitaciones sefialadas al comienzo de esta revisión. A nuestro juicio, la información revisada señala, por una parte, que la aplicación de dichos métodos de djagnóstico es del todo deseable cuando la eva. luación de pacientes con ITU involucra objeti. vos de investigación, pero se requiere de más información, en nuevos estudios, para decidir sobre las ventajas que of recen sobre los métodos clásicos, en la investigación rutinaria de la localjzación anatómica de la ITU. La medición de enzimas en la orina, cuyo valor es aún controvertido, se insinúa como una alternativa atractjva, particularmente en términos de simplicidad y costo.

Se ha avanzado de manera significativa en la comprensión de los factores que participan en la interacción entre el huésped y el agente infeccioso, cuyo resultado determina la forma de presentación de la enfermedad. El conocimiento alcanzado respecto del principal factor de virulencia de Escherichia coli para la vía urinaria, la fumbria $P$, permite suponer que en los próximos af̂os se intensificará la investigación, ya inicia. da, en tomo al desarrollo de vacunas para prevenir la ITU.

\section{Resumen}

Durante los últimos 10 affos se han incorporado nuevos conceptos al conocimiento de ja patogenia de las infecciones del tracto urinario (ITU), y se han perfeccionado métodos de estudio que permiten mejor valoración clínica de los pacientes con estas afecciones. En esta revisión se analiza la información relativa a factores de la virulencia de $E$. coli en el tracto urinario y sobre marcadores genéticos del huésped como posibles indicadores de riesgo de recurrencia y complicaciones. También se revisaron algunas de las posibilidades que ofrecen, en el terreno clinico, los procedimientos diag. nósticos recientemente propuestos para determinar el nivel anatómico de la infección.

(Palabras clave: infección del tracto urinario, $E$. coli, factores de virulencia, factores del huésped.)

\section{Referencias}

1. Winberg, J.; Jacobson, $S$,; Eklof, O, et al.: Development of hipertension and uremia following childhood pyelonephritis. 30 years follow up. Abstract 4005: VIII Congress of the International Pediatric Nephrology Association. Toronto, AugustSept. 1989.

2. Wimberg, J.; Bollgren, I.: Kallenius, C. et al.: Clínical pyelonephritis and focal renal scarring. A selected review of pathogenesis, prevention and prognosis. Ped Clin N Am 1982; 29: 801-814. 
3. Winter, A.L.; Hardy, B.E. Aiton, D.J. et al.: Adquired renal scarss in children. J Urol 1983 ; 129: $1190-1194$.

4. Marild, S; Hellstrōm, M.; Jacobsen, B. et al.: Kidney size and growth in relation to adquisition of renal damage following acute childhood pyelonephritis. Abstract 21.007: VIII Congress of the International Pediatric Nephrology Association. Toronto, August-Sept. 1989.

5. Neumonn, C.G.; Pryles, C.V.: Pyelonephritis in infants and children. Autopsy experiency at the Boston City Hospital 1933-1960. Am J Dis Child $1962 ; 104: 215-229$.

6. Gillenwater, J.Y.; Harison, R.B.; Kunin, C.M.: Natural history of bacteriuria in school girls. A long term case-control follow up. $N$ Engl J Med $1979 ; 301: 396-399$.

7. Pylkkanen, J.; Vilska, J., Koskimies, O.: The value of level diagnosis of childhood urinary tract infections. Acta Pediats Scand 1981; 70: 879 . 893.

8. Sheldon, C.A.; Gonzales, R.: Differentiation of upper and lower urinary tract infections: How and when? Med Clin North Am 1984; 68: 321333.

9. Jodal, U.; Wirberg, I.: Management of children unobstructed urinary tract infection. Ped Nephrol $1987 ; 1: 647-656$.

10. Miller, $T_{*}$; Phillips, $S$ : Pyelonephritis: the relationship between infection, renal scarring and antimicrobial therapy. Kid Jnt 1981; 19: 652-654.

11. Slotki, I.N.; Assher, A.W.: Prevention of scarring in experimental pyejonephritis in the rat by early antibiotic therapy. Nephron 1982; 30: 262-268.

12. Smellie, I.M,; Ramsley, P.G.; Nomand, I.C.S. ef al.: Development of new scars: A collaborative study, Br Med J 1985; 290 : 1957-1960.

13. Berg, U.B.; Johensson, S.B.: Age as a main determinant of renal functional damage in urinary tract infection. Arch Dis Child 1983; 58: $963-$ 969.

14. Aronson, A.S.; Svenningsen, N.W.: DDAVP for estimation of renal concentrating capacity in infants and children. Arch Dis Child 1974; 49: 654-659.

15. Risdon, R.A; Parkhouse, H.F.; Godley, M.L. et al.: 99MTechnetium-Dimercaptosuccinic acid renal imaging in the detection of acute pyelonephritis. Abstract \$3-01. Presentado a: VIII Congress of International Pediatric Nephrology Association. Toronto, August-Sept., 1989.

16. Wikstad, I,; Hannerz, L.; Karlsson, A.; Eklof, A.-Ch.; Oling, S.; Aperio, A.: 99MTechnetiumDimercaptosuccinic acid scintigraphy in the diggnosis of acute pyelonephritis in rats Pediatr Nephrol $1990 ; 4: 331-334$.

13. Tappin, D.M.; Murphy, A.V.; Mocan, H. et al.: A prospective study of children with first acutc symptomatic $E$. coli urinary tract infection. Early 99 Technetium Dimercaptosuccinic acid scan appearances. Acta Paediatr Scand; 1989; 78: 923.929 .

18. Rosemberg, A.R.; Wilson, M.F,; Rossieigh, M.A.; Elison, B.; Cajil, S.; Khill, S.; Farnsworth. R.H.: DMSA Scanning in infants - A Retrospective
Study. Abstract 21.004: VIIl Congress of the International Pediatric Nephrology Association. Toronto, August-Sept. 1989.

19. Johnson, C.E.; Vacca, Ch.U.; Fatlar, D.: Urinary N-acetyl-B-Glucosaminidase and the selection of children for Radiologic Evaluation After Urinary Tract Infection. Ped 1990;86: 211-216.

20. Vigano, A.; Assael, B.M.; Dallo Villo, A. at al.: $\mathrm{N}$-Acetyl-B-D-Glucosaminidase (NAG) and NAG Isoenzymes in children with upper and lower urinary tract infections. Clin Chim Acta 1983; 297-304.

21. Jewkes, F.E.M.; Gupta, S.C.: Price, D.A,; Houston, I.B.; Postlethwaite, R.J.: Evaluation of Dinetcaptosuccinic acid scanning and measurement of urinary proteins in the diagnosis of upper arinary tract infection. Abstract 21.003: VIII Congress of the International Pediatric Nephrology Association. Toronto, August-Sept. 1989.

22. Lindin-Janson, G.; Hanson, L.A.; Kaijer, B. et al.: Comparisson of Escherichia coli from bacteriuric patients with those from facces of healthy school children. J Infect Dis $1977 ; 163: 346-353$.

23. Ollin, S.; Hanson, L.A.; Holmgren, J, et al.: The bactericidal effect of normal human serum on $E$. coli from normals and from patients with UTI. Infection $1973 ; 1: 24-28$.

24. Atchman, M.; Kusecek, B. et ol. : Six widespread bacterial clones among Eschechiria coli $\mathbf{K} 1$ isolates, lnfect Immun 1983; 39: 315-335.

25. Vatsanen-Rhen, $V_{*}$ Elo, $J_{\text {; }}$ Vaisanen, $E$, et al.: P-fimbriated clones among uropathogenjc Escherichia coli strajns. Infect Immun 1984; 43: 149155.

26. Orskov, F,; Orskov, $I$ : Summary of a workshop on the clone concept in the epidemiology, taxonomy and evolution of the Enterobacteriaceae and other bacteria. J Infect Dis 1983 ; 148: 346357.

27. Caugant, $D A$.: Enzime polymorphism in Excherichia coli: Genetjc structure of intestinal populations: Relationship with urinary tract infection strains and with Shighella. Academic Thesis, University of Goteborg, Coteborg, Sweden, 1983.

28. Caugant, D.A.; Levin, B.R.; Lindin-Janson, $G$. et al.: Genetic diversity and relationships among strains of Escherichia coll in the intestine and those causing urinary tract infections. Progr Allergy $1983 ; 33: 203-227$.

29. Orskov, I.; Suanborg, E.C.; Orskov, F.: Aerobactin production of serotyped Escherichio coli from urinary tract infections. Med Microbiol Inmunol $1988 ; 177: 9-14$.

30. Jacobson, S.H.; Hammarlind, M.; Liderfeldt, $J . K$. et al.: Incidence of aerobactin positive Esche. richia coli strains in patients with symptomatic Urinary Tract Infection. Eur J Clin Microbiol Infec Dis 1988; $7: 630-634$.

31. Svanborg, E, Hanson, L.A.; Jodol, $U$, et al.: Variable adherence to normal urinary tract epithelia] cells of Escherichio coli strains associated with various forms of urinary tract infections. Lancet $1976 ; 2: 490-492$.

32. Svanbory, E.; Ericksson, B.; Henson, L.A. et al.: Adhesion to normal human uroepithelial cells of 
$E$. coll of children with various forms of urinars tract infections. J Pedjatt 1978; 93: 398-403.

33. Kallenius, G.; Svensson, S.B.; Hultberg, H.: Oc cursence of P-fimbriated Escherichia coli in urinary tract infections. Lancet $1981 ; 2: 1369-1372$.

34. Stenqvist $K_{+i}$ Sandberg, T.; Lindin-Janson, $G$. et al: Virulence factors of $E$. coli urinary isolates from pregnant women, J Infect Dis 1987; 156: 870-877.

35. Sandberg, $T$; Kaisjer, B.; Lindin-Janson, $K$. ef al.: Virulence of Escherichto coli in relation to host factors in women with symptomatic urinary tract infections. J Clin Microbiol 1988; 26: 1471-1476.

36. Arthur, M.; Johnson, C.E.; Rubin, R.H. et al.: Molecular epidemiology of adhesin and hemolisin virulence factors among uropathogenic Escheri. chia coli. Infect Immun 1989;57: 303-313

37. Arthur, M.; Companelli, C; Arbeit, R.D. et al. Structure and copy number of gene clusters related to the pap Pathesine operon of uropathogenic Escherichia coli. Infect Immun 1989; 57: 314-321.

38. Lefler, H.; Suanborg, E.C.: Chemical identification of a gly cosphingolipid receptor for Escherichia coli attachirg to human epithelial cells and agglutinating erythrocytes. FEMS Microbiol Letter 1981; 8: 127-134.

39. Kallentus, G.; Svensson, S.B. et aI.: The pk antigen as receptor for the haemagglutination of pyelonephritic $E$. colt. FEMS Microbiol Letter 1980; 7: 297-302.

40. Vaisanen, $D$. and other. (Letter) Blood group $M$ specific haemagglutin in pyelonephritogenic $E_{s}$ cherichio coli. Lancet 1982;1: 1192.

41. Lomberg, H.; De Man, P,; Svanbory, C.: Bacterial and host determinants of renal scarring. APMIS 1989; 97: 193-199.

42. Lomberg, H.; Hellström, M.i Jodal, U. et al. Properties of Escherichia coll in patients with renal scarting. J Infect Dis $1989 ; 159: 579-581$.

43. Morlid, $S_{\text {.; }}$ Wettergren, B.; Hellstróm, M. et ol.: Bacterial virulence and inflammatory response in infants with febrile urinary tract infection or screening bacteriuria, $J$ Pediatr 1988; 112 : 348-354

44. De Man, $P$,; Jodal, $U$.; Lincond, $X$, et ol: : Bacterial attichment and inflammation in the urinary tract. J Infect Dis 1988; $1: 29-35$.

45. De Man, P.; Claeson, I,; Johonson, I et al.: Bacterial attachment as a predictor of renal abnormalities in boys with urinary tract infections. J Pediatr $1989 ; 115: 915-922$.

46. Marid, S.; Hellström, M.; Sodal, $U$, et al.: Proceedings of the IVth International Symposium on Pyetonephritis. 1986, p. 58.

47. Elo, $J_{;}$Tallgren, $V_{\text {,; }}$ Voisanen, $T$, et al: : Association of $\mathbf{P}$ and other fimbriae with clinical pyelo- nephritis in children. Scand J Urol Nephrol 1985; 19: $281-284$

48. Kaack, M.B.; Per, A.; Korhonen, T K.; Roberts, J.A.: P.fimbriae yaccines. I. Cross reactive protection against pyelonephritis. Pediatr Nephrol 1989:3: 386-390.

49. Raberts, J.A.; Kaack, M.B.; Baskin, G.; Korhonen, T.K.; Svenssan, S.B.; P-fimbriae vaccines. II. Cross reactive protection against pyelonephritis. Pedilatr Nephrol $1989 ; 3 ; 391-396$

50. Archambaud, M.; Courcoux, P.; Ouin, V.; Chabanon, $G$, et al.: Phenotypic and genotypic assay for the detection and identification of adhesines from pyelonephritic Escherichia coli. Ann Inst Pasteur Microbiologio 1988; 139: 557-573.

51. Labigne-Roussel, A.; Folkow, S.: Distribution and degree of heterogeneity of the fimbrial adhesineencoding operon (afa) among uropathogenic Escherichia coll isolates. Infect Immun 1988; 56: 640-648.

52. Labigne-Roussel, A.; Schmidr, M.A.; Waltz, W. et al.: Genetic organization of the afimbrial adhesine operon and nucleotide sequence from a urophatogenic Escherichio coli gene encoding an afimbrial adhesine. J Bacteriol 1985; 162: 1285 1292.

53. Labigne-Roussel, A.: Lark, $D$.; Schoolnick, $G$ et al.: Clonning and expression of an afimbrial adhesine (AFA-I) responsible for $P$ blood groupindependent, mannose-resistant haemagglutination from a pyelonephritic Escherichia coli strains. Infect Immun 1984;46: 251-259.

54. Cruz-Coke, R.; Paredes, L.; Montenegro, A.: Blood groups and urinary microrganism. J Med Genet 1965;2: 185-188.

55. Radner, J.J; Thomos, V.L.; Fortand, $M$.: Relationships between human blood groups, bacterial pathogens and urinary tract infections. Am J Med Sci 1986; 292:87.91.

56. Lomberg, $H_{\text {.; }}$ Cedergren, $B_{\text {; }}$ Leffler. $H$. et al. Intluence of blood group on the availability of receptors for attachment of uropathogenic $E_{s-}$ cherichio coli. Infect Immun 1986; 51: 919-926.

57. Tomisawd, $S$,; Kogure, $T$; Kuroume, $T$, er al. p-blood group proneness to urinary tract infections in japanese children. Scand J Infect Dis 1989; 4: $403-408$

58. Blackwell, C.C.; May, S.J.; Brettle, R.P et al. Secretor state and immunoglobuline levels amons women with recurrent urinary tract infections. J Clin Lab Immunol 1987; 22: 133-137.

59. Stamm, W.E., Hooton, T.M.; Johnson, J.E. et al. Urinary tract infections from pathogenesis to treatment. I Infect Dis 1989; 159: 400-406. 